\title{
Noisy Voltage Sag Signal Analysis Based on Wavelet Transformation
}

\author{
Min $\mathrm{Cao}^{1,2, a}$, Shilin $\mathrm{Li}^{2, \mathrm{~b}}$, Qingchan $\mathrm{Liu}^{2, \mathrm{c}}$ \\ Chong $\operatorname{Lin}^{2, d}$, Zhiqiang $\mathrm{Ma}^{3, \mathrm{e}^{*}}$ \\ ${ }^{1}$ Yunnan Electric Power Research Institute, Kunming65027, China \\ ${ }^{2}$ Key Laboratory of CSG for Electric Power Measurement, Kunming650217, China \\ ${ }^{3}$ School of Electrical Engineering, Chongqing University, Chongqing 400030, China. \\ acm1961@sohu.com, b 497958071@qq.com, ' 21902151@qq.com, \\ d371223910@qq.com , e851231278@qq.com
}

Keywords: Wavelet Transformation; Voltage Sag; FIR filter; soft-threshold de-noising.

Abstract. In order to detect the starting and recovery points of noisy voltage sag signal, this paper proposed the wavelet soft threshold method for denoising and the wavelet transform for detecting the singular points. Comparing to the FIR filtering method, the wavelet soft threshold method can filter out the high-frequency and low-frequency noise component simultaneously. The simulation results show that the wavelet soft threshold method achieves a higher signal-to-noise ratio (SNR) and smaller root mean square error (RMSE). After denoising processing, the wavelet method can identify the starting and recovery points of sag signal accurately.

\section{Introduction}

Currently, increasing attentions are attracted to power quality problems; especially the voltage sags cause greater dangers. It has become the focus of transient power quality problems. There are many methods for detecting voltage sag. However, considering the short duration and great uncertainty, traditional methods, as Fourier transforms can accurately detect the fundamental amplitude. However, its response is slow cannot detect the voltage sag real-time. Others can be divided into two categories generally, the time-based and the transform-based methods. The former includes the RMS voltage, the peak voltage, the defect voltage and etc. The latter includes the dq transform, wavelet transform, the Hilbert-Huang transform (HHT), the short time Fourier transform (STFT), etc [1].

Among above methods, the wavelet transform method has good time-frequency locality and multi-resolution property. It shows obvious advantageous in analyzing transient and mutation signals. The scaling and translation operations of wavelets achieve multi-scale analysis, which is a complementary for Fourier method for detecting short time disturbances in power quality analysis. Therefore, it is widely used and has become an important tool for transient power quality analysis [2].

Considering the external environment factors as electromagnetic interference, the detected voltage sag signals contains noise. And the noisy components will affect the wavelet method performance. Hence, this paper proposed the improved wavelet based distortion detection method for noisy signal voltage sag. The main idea is based on the following facts that the noisy component is mainly corresponding to the high frequency part in wavelet decomposition, the noise can be weakened and filtered by threshold process and the distorting can be detected after denoising. The simulation results show that the algorithm maintains high accuracy and reliability even in strong noise situations.

\section{Organization of the Text}

The Principle of Wavelet Analysis Algorithm. The wavelet transform is defined by the wavelet function and scale function [3]. For continuous function $\mathrm{f}(\mathrm{t})$, it can be expanded as: 


$$
\begin{aligned}
f(t) & =\frac{1}{C_{\psi}} \int_{0}^{+\infty} \frac{d a}{a^{2}} \int_{-\infty}^{+\infty} W T_{f}(a, \tau) \Psi_{a, \tau}(t) d \tau \\
& =\frac{1}{C_{\psi}} \int_{0}^{+\infty} \frac{d a}{a^{2}} \int_{-\infty}^{+\infty} W T_{f}(a, \tau) \frac{1}{\sqrt{a}} \psi\left(\frac{t-\tau}{a}\right) d \tau .
\end{aligned}
$$

Wherein, $W T_{f}(a, \tau)$ is known as the transform coefficients or wavelet transform formula as:

$$
W T_{f}(a, \tau)=<f(t), \psi_{a, \tau}(t)>=\frac{1}{\sqrt{a}} \int_{R} f(t) \psi *\left(\frac{t-\tau}{a}\right) d t .
$$

The wavelet function:

$$
\psi_{a, \tau}(t)=|a|^{-\frac{1}{2}} \psi\left(\frac{t-\tau}{a}\right)
$$

Can be discretized by the scale and translation parameters, then forming the discrete wavelet transform:

$$
W T_{f}\left(a_{0}^{j}, k \tau_{0}\right)=\int f(t) \psi_{a_{0}^{j}, k \tau_{0}}^{*}(t) d t, j=0,1, \mathrm{~L}, \quad k \in Z
$$

The discrete wavelet expanded has:

$$
f(t)=\sum<f, \psi_{j, k}>\cdot \psi_{j, k}(t)=\frac{1}{A} \sum_{j, k} W T_{f}(j, k) \psi_{j, k}(t) .
$$

According to the Multi-resolution analysis theory, any function in the space V0, for example, the signal S can be decomposed into a low frequency part A1 and a high frequency part D1; then the low frequency part A1 can be further decomposed into A2 and D2; repeating the above steps then we can obtain An and Dn , $n \in Z$. The multi-resolution analysis framework is listed as Fig. 1:

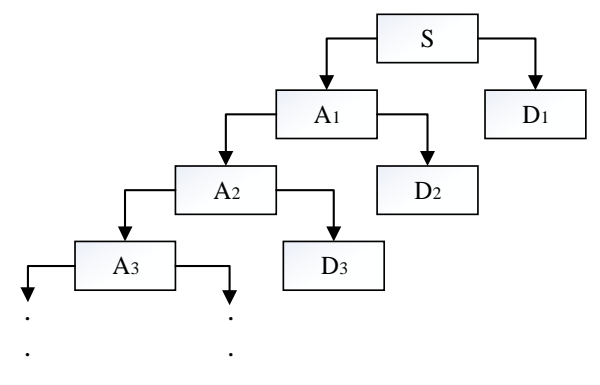

Fig.1. Multi-resolution analysis chart

Generally, increasing the decomposition scale, the wavelet coefficient for signal will increase, while for the noise will decrease. Therefore, the signal can be decomposed continually in wavelet space, and the disturbances or mutations can be detected by at appropriate hierarchical scale. However, there are still some drawbacks as [4]: firstly, it needs multi-wavelet decompositions and will be huge time-consuming. Then, the decomposition level is limited for specific sampling frequency. Finally, for big noise intensity situation, the disturbance cannot be detected accurately.

Therefore, de-noising process is necessary for signal with noise. The original signal is distributed in the low-frequency, while the noise disturbance is in high-frequency. In the wavelet transform, the signal energy and the noise energy will be distributed uniformly in every level. But the wavelet coefficients of the signal have greater amplitude, which the coefficients of noise are relatively small. Thus, forming a threshold de-noising method in wavelet analysis, the basic idea is setting a threshold value for each layer and comparing the threshold value and wavelet coefficients.Finally, using the processed wavelet coefficients, the filtered signal can be reconstructed and the noise is weakened.

It's obvious that the threshold value selection is the key factors in de-noising processing. The easiest selection is setting the threshold as constant and called the hard threshold method. Yet, it is 
difficult to apply in practical situations because of the lack of flexibility [5]. Considering the randomness of the noise intensity and the differences of propagation characteristics, the threshold values should be dynamically changed with the coefficients, namely, the soft threshold method [6]. The common threshold function expression is:

$$
\hat{W}_{j, k}= \begin{cases}\operatorname{sgn}\left(W_{j, k}\right)\left(\left|W_{j, k}\right|-\lambda\right) & \left|W_{j, k}\right| \geq \lambda \\ 0 & \left|W_{j, k}\right| \leq \lambda .\end{cases}
$$

Because the soft threshold function has good continuity, the larger wavelet coefficients are greatly reduced in de-noising process. So the reconstructed waveform is much smoother.

Analysis of Voltage Sags Signal with Noise. The voltage sag is defined as: the voltage root mean square (RMS) value decreases to $0.1 \sim 0.9 \mathrm{pu}$ with duration of 0.5 cycles to $1 \mathrm{~min}$. The voltage sag signal is shown in fig. 2. Its RMS is $220 \mathrm{~V}$, and frequency is $50 \mathrm{~Hz}$, the sampling frequency is $6.4 \mathrm{kHz}$, so corresponding to 128 sampling points in every cycle. The signal duration is $0.7 \mathrm{~s}$. The voltage sag occurs at $0.2 \mathrm{~s}$ with recovering at $0.4 \mathrm{~s}$. For noisy situation, the noise is superimposed in signal with signal-to-noise ratio (SNR) is $35 \mathrm{~dB}$. The signal model is as Eq. 7 and Fig. 2:

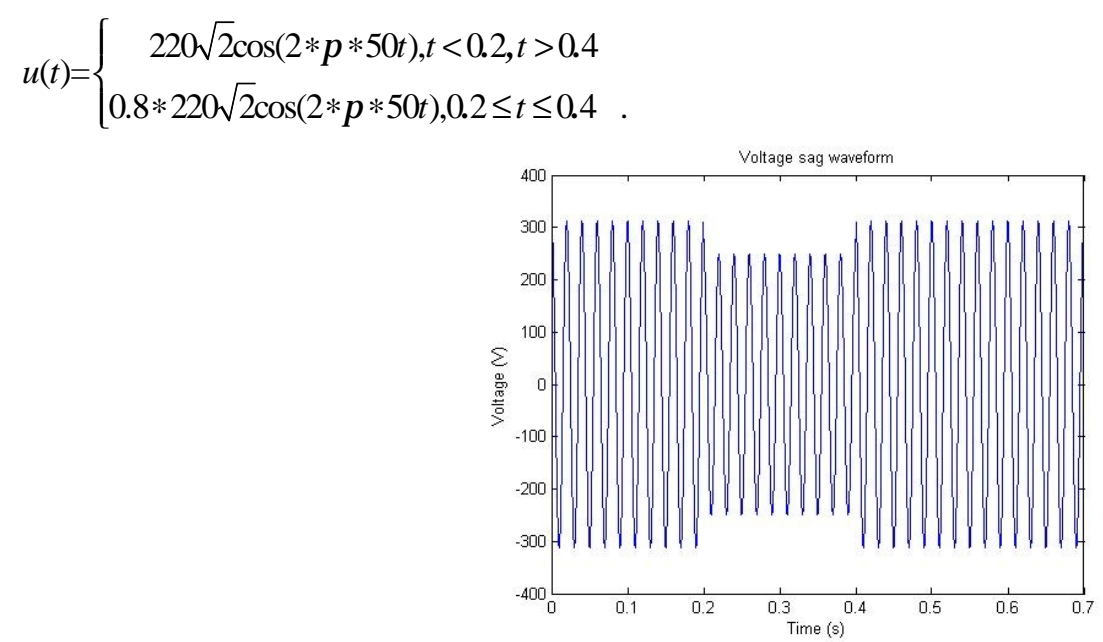

Fig.2. Original voltage sag signal

Singularity Detection For Original and Noisy Signal. The original signal is decomposed by "db4" wavelet basis in three-scale and the first level reconstructed detail coefficients are shown in Fig. 3. It can be seen that the "db4" wavelet can detect and identify the starting and recovery points effectively. Similarly, using the "db4" wavelet basis decompose the noise single in the same scale and obtaining the corresponding reconstructed detail coefficients as Fig. 4. It can be seen: owing to the noise effect, the wavelet can't effectively identify the starting and recovery points.
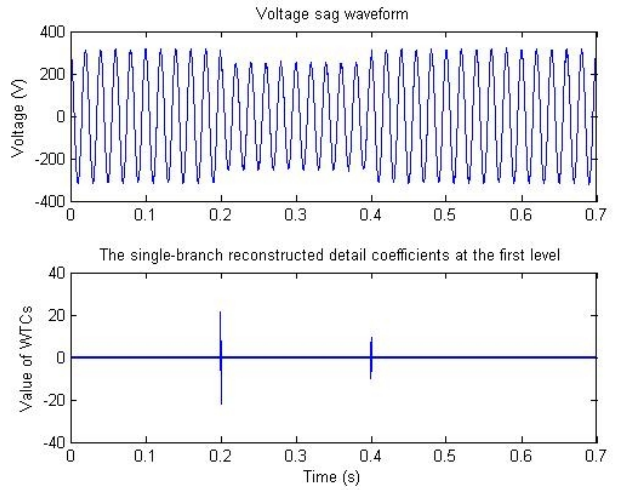

Fig.3. Original voltage sag signal and its first reconstructed detail coefficients
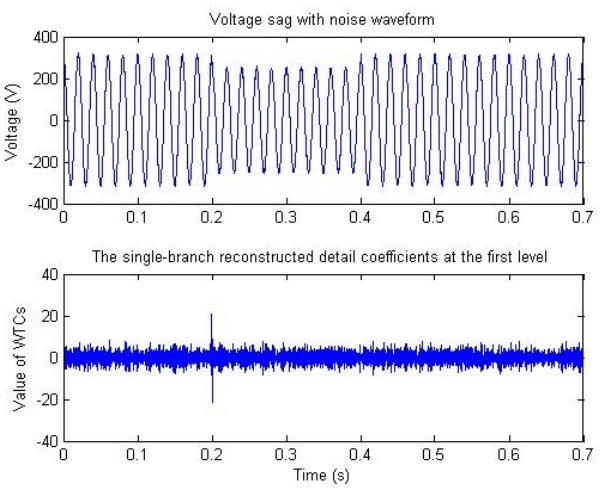

Fig.4. Noisy voltage sag signal and its first level evel reconstructed detail coefficients 
In the direct decomposition processing, the original signal and noise component will both be retained. Thus, the singularity points can be accurately determined and a preprocessing of de-noising is needed.

FIR Filtering Processes of Noisy Signal. Firstly, the noise is filtered by the FIR filter, and then using the db4 wavelet basis decompose the filtered signal. Because the original signal frequency is $50 \mathrm{~Hz}$, the cutoff frequency of FIR low pass filter can be set at $80 \mathrm{~Hz}$. The corresponding digital frequency of FIR filter is 0.025. Its amplitude-frequency response is shown in Fig. 5.

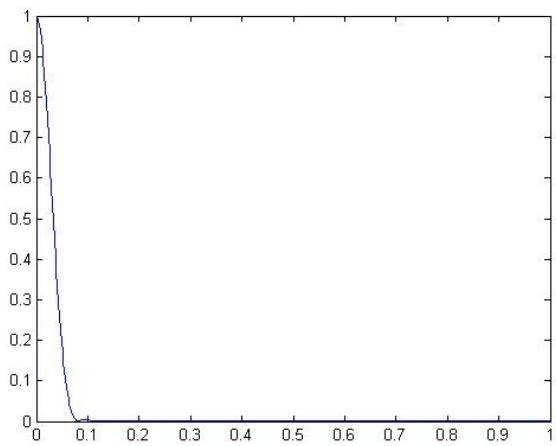

Fig.5. Amplitude-frequency response of FIR filter
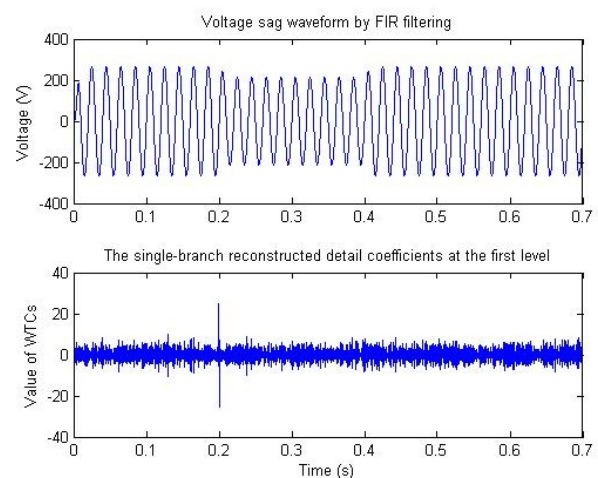

Fig.6. FIR filtered signal and its first level reconstructed detail coefficients

The noisy signal is filtered by above FIR filter, performed the wavelet decomposition and the singular point detection, as shown in Fig. 6.It can be seen that the wavelet method still cannot identify the singularity. The reason is that: although the FIR filter can remove the high frequency noise, the low frequency noise is still retained. While in the wavelet decomposition, the low frequency noise still affects the identification of the starting and recovery points.

Wavelet Soft Threshold Method For Noisy Signal. Then, the noisy signal is filtered by the wavelet soft threshold method, performed the wavelet decomposition and the singular point detection, as shown in Fig. 7. We can find that: comparing to the FIR filtering, the wavelet soft threshold method can simultaneously filter out the noise of every frequency band. Then the modulus maximum algorithm can detect the singularity points accurately.
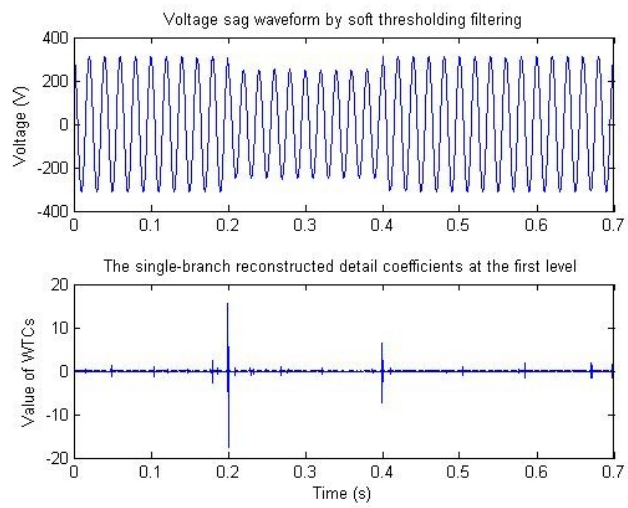

Fig.7. Wavelet soft threshold filtering processing and its first level reconstructed detail coefficients

Comparison of De-Noising Effect. In order to analyze the de-noising performance, two indices as SNR and root mean square error (RMSE) were adopted [7]. The SNR and RMSE are defined respectively as:

$$
\mathrm{SNR}=10 \lg \left(\frac{\sum_{j=1}^{N} x^{2}(j)}{\sum_{j=1}^{n}[x(j)-\hat{x}(j)]^{2}}\right)
$$




$$
R M S E=\left[\frac{1}{N} \sum_{j=1}^{n}(x(j)-\hat{x}(j))^{2}\right]^{1 / 2}
$$

where $\mathrm{x}(\mathrm{j})$ is the amplitude of the original signal at the sampling point $\mathrm{j}, \hat{x}(j)$ is the filtered signal at the sampling point $\mathrm{j}, \mathrm{N}$ is the signal length. In above two indexes, the larger SNR and smaller RMSE mean better de-noising performance.

Table.1 De-noising comparison in different SNR

\begin{tabular}{ccl}
\hline Before filtering & After filtering & RMSE \\
\hline $35[\mathrm{~dB}]$ & $43.4132[\mathrm{~dB}]$ & 1.407 \\
$40[\mathrm{~dB}]$ & $47.8158[\mathrm{~dB}]$ & 0.8473 \\
$45[\mathrm{~dB}]$ & $52.5517[\mathrm{~dB}]$ & 0.4913 \\
$50[\mathrm{~dB}]$ & $57.4832[\mathrm{~dB}]$ & 0.2785 \\
\hline
\end{tabular}

As can be seen from Table. 1 that: after de-noising processing, the SNR gets larger and the RMSE gets smaller by the wavelet soft threshold method.

\section{Conclusion}

Considering the noise in actual measurement, the performance of wavelet will be affected and even invalid when detecting voltage sag directly. Therefore, this paper proposed the wavelet soft-threshold method for de-noising and then using wavelet transform in detecting the singular points. The simulations show that: comparing to the FIR filtering, the wavelet soft threshold method can filter out high-frequency and low-frequency noise simultaneously, thus it can detect and identify the singular points of voltage sag with noise.

\section{Reference}

[1] Dong Na. Research on the rapid detection methods of voltage sag [D], HuNan University, 2013. In Chinese.

[2] Tan ping. Test and Location of Power Quality Disturbances by Wavelet Transformation [J], Electrotechnics Electric, 2010, 06: 54-57. In Chinese.

[3] Peng Yuhua, Wavelet Transform and engineering applications. Beijing: Science Press, 1999, 13-44. In Chinese.

[4] Ouyang sen, Song Zhongxiang, Chen Degui, Wang Jianghua. Application of wavelet soft-threshold de-noising technique to power quality detection Automation of Electric Power Systems, 2002, 26(19): 56-60. In Chinese.

[5] Santoso S, Powers E J, Grady W M. Power Quality Disturbance Data Compression using wavelet transform methods [J]. IEEE Trans on Power Delivery, 1997, 12(3): 1250 1257.

[6] Donoho D L. De-noising by Soft thresholding. IEEE Trans on Information Theory, 1995, 41(3):613 627.

[7] KeHui, GuJie. Wavelet Threshold De-noising of Power System Signals. Proceedings of the CSEE, 2010, 02:103-108. In Chinese. 